\title{
Quantum Fourier-Transform Infrared Spectroscopy for Complex Transmittance Measurements
}

\section{$\operatorname{AUTHOR}(\mathrm{S}):$}

Mukai, Y.; Arahata, M.; Tashima, T.; Okamoto, R.; Takeuchi, S.

\section{CITATION:}

Mukai, Y. ...[et al]. Quantum Fourier-Transform Infrared Spectroscopy for Complex Transmittance Measurements. Physical Review Applied 2021, 15(3): 034019.

\section{ISSUE DATE:}

2021-03

URL:

http://hdl.handle.net/2433/261908

\section{RIGHT:}

(c) 2021 American Physical Society; 許諾条件に基づいて掲載しています $\circ$ 


\title{
Quantum Fourier-Transform Infrared Spectroscopy for Complex Transmittance Measurements
}

\author{
Y. Mukai®,$^{1}$ M. Arahata $\odot,{ }^{1}$ T. Tashima $\odot,{ }^{1}$ R. Okamoto $\odot,{ }^{1,2}$ and S. Takeuchi ${ }^{1, *}$ \\ ${ }^{1}$ Department of Electronic Science and Engineering, Kyoto University, Kyotodaigakukatsura, Nishikyo-ku, Kyoto \\ 615-8510, Japan \\ ${ }^{2}$ Japan Science and Technology Agency, PRESTO, Gobancho, Chiyoda-ku, Tokyo 102-0076, Japan
}

(Received 27 September 2020; revised 23 December 2020; accepted 29 January 2021; published 8 March 2021)

\begin{abstract}
Harnessing the quantum interference of the pair generation processes, infrared quantum spectroscopy, based on nonlinear interferometers with visible-infrared photon-pair sources, enables the extraction of the infrared optical properties of a sample through visible photon detection without the need for an infrared optical source or detector. We develop a theoretical framework for quantum Fourier-transform infrared (QFTIR) spectroscopy. The proposed Fourier analysis method, which fully utilizes the phase information in the interferogram, allows us to determine the complex transmittance and optical constants for a sample in a simple setup without the use of any dispersive optics for spectral selection. In the experimental demonstrations, the transmittance spectrum of a bandpass filter and the refractive index of silica glass are measured in the near-infrared region using QFTIR operated in a low gain regime; these results agree well with the independently measured spectrum using a conventional spectrometer and an value estimated from references. These demonstrations prove the validity and great potential of QFTIR spectroscopy.
\end{abstract}

DOI: 10.1103/PhysRevApplied.15.034019

\section{INTRODUCTION}

Advances in infrared spectroscopic techniques over the past half-century have made it possible to perform fast, nondestructive material identification and molecular recognition. Infrared spectroscopy has become an indispensable tool in both science and industry, finding numerous applications, such as product inspection, environmental sensing, and security checking [1]. However, conventional infrared spectrometry has technical difficulties owing to the low sensitivity of detectors and low efficiency of light sources in the infrared region.

In the last decade, the field of photonic quantum technologies has been growing rapidly. Various types of entangled photon sources have been investigated [2] and applied to quantum information [3-7] and sensing [8-14]. Among them, nonlinear interferometers, consisting of multiple entangled-photon sources, as demonstrated by Mandel and his collaborators [15], have been attracting considerable attention [16] and, for example, used for "quantum imaging with undetected photons" [12]. It should be noted that these experiments performed in a so-called "low gain regime," where the average photon flux is below one photon pair per correlation time of the biphotons, cannot be described by classical theory and should be understood as

\footnotetext{
*takeuchi@kuee.kyoto-u.ac.jp
}

a result of the quantum interference of the pair generation processes $[17,18]$.

Recently, infrared quantum absorption spectroscopy (IRQAS) techniques based on nonlinear interferometers operated in a low gain regime were proposed and demonstrated by Kalashnikov et al. [19,20]. In this method, information on optical loss and/or phase retardation of infrared photons, caused by interactions with a sample of interest, can be converted to a generation probability for correlated visible photons [21,22]. This feature of IRQAS allows us to extract infrared optical properties of the sample, requiring only a visible light source and detectors.

In the actual implementation of IRQAS, it is important to spectrally resolve the visible photon spectrum and quantitatively evaluate the visibility of interferograms at each wavelength to obtain the IR absorption spectrum. Since photon-pair sources, usually based on spontaneous parametric down-conversion (SPDC) in optical nonlinear crystals [23], emit an infrared-visible photon pair with a finite bandwidth, a measured interferogram is spectrally averaged if there is no spectral filtering component. The most straightforward way to get a spectrally resolved interferogram is to use dispersive optics for visible light to spread its spectral components in space, as experimentally demonstrated using a spectrograph [19] or monochromator [20].

Another possible approach is to apply Fourier analysis to the nonlinear interferometric signal. Very recently, 
Lindner et al. have experimentally demonstrated that an infrared spectrum can be inferred from a Fourier analysis of interferograms measured with a nonlinear Michelson interferometer [24]. In their experiment, the visible photon count rate was measured as a function of the optical path length difference between the interferometer arms, which exhibited an interferometric fringe pattern. In their experiment, the transmission spectra were obtained using Fourier transformed interferograms with and without a sample (polypropylene film) for the wavelength range 3.2-3.9 $\mu \mathrm{m}$. The results agreed well with data obtained by conventional FTIR spectroscopy. However, while they presented the procedure using the Fourier transformed interferogram, the theoretical background for the procedure was not provided. Related to this, the analysis was limited to the magnitude of the transmittance, and the ability to obtain the complex transmittance of the sample was not discussed.

In the present study, we develop a theoretical framework for QFTIR spectroscopy, which provides a clear procedure to determine the infrared spectrum via Fourier analysis of the interferogram obtained with a nonlinear interferometer operated in a low gain regime (hereinafter called a "quantum interferogram"). Similar to conventional FTIR spectroscopy, QFTIR spectroscopy has advantages compared to dispersive (scanning) spectroscopy, such as a high throughput, multiplexing, and wave number accuracy $[1,25,26]$. In addition, direct measurement of the phase information in a Fourier spectrum allows us to determine the complex transmittance and, consequently, to calculate the complex-valued optical constants for a sample, such as the refractive index, without using a Kramers-Kronig transformation. This is highly beneficial for material characterization in the infrared region, as is well known in time-domain spectroscopy $[27,28]$. We also build a nonlinear interferometer to experimentally realize a QFTIR spectroscopy system and demonstrate a transmittance measurement for a bandpass filter in the near-infrared region, at around $1550 \mathrm{~nm}$; the results show good agreement with an independently measured spectrum using a conventional spectrometer. We also measure a complex transmittance for silica glass and calculate its complex refractive index fully utilizing the phase information of the quantum interferogram. The obtained value for the refractive index coincides with the literature value. Since the size of the IR light source and IR detector have been obstacles to the miniaturization of conventional FTIR, QFTIR requiring only a visible light source and detectors has strong potential as a compact and cost-effective IR spectroscopy system. These results prove the validity and great potential of QFTIR spectroscopy.

\section{THEORY FOR QFTIR SPECTROSCOPY}

In this section, we provide a theoretical basis for QFTIR spectroscopy. First, we derive an analytical expression for the quantum interferogram measured with a nonlinear interferometer, that is, the count rate for signal photons recorded as a function of the optical path length difference between the signal and idler photons. Second, we show that the Fourier transform of a quantum interferogram gives information about the complex transmission coefficient for the sample, from which the complex refractive index can be directly calculated.

\section{A. Analytical expression for quantum interferogram}

Let us consider a nonlinear Michelson interferometer, schematically shown in Fig. 1(a). In this setup, a monochromatic pump light with a frequency $\omega_{p}$ passes through a nonlinear optical crystal twice, triggering two consecutive photon-pair generation processes. In the first passage through the crystal, the pump photon is converted into a photon pair via SPDC. Energy conservation requires a correlation between the signal photon frequency, $\omega_{s}$, and the idler photon frequency, $\omega_{i}$, such that their sum equals that of the pump photon, $\omega_{p}=\omega_{s}+\omega_{i}$ [29]. Suppose that the phase matching condition for SPDC is satisfied for visible (signal)-infrared (idler) photon-pair generation. The pump and signal photons are reflected by a dichroic mirror and propagate together along an interferometer arm. They are reflected back to the nonlinear crystal by an end mirror $\mathrm{M}_{s}$. The optical path length of this interferometer arm is fixed at $L_{s}$. The idler photon transmitted by the dichroic mirror, spatially separated from the pump and signal photons, is sent into the other arm of the interferometer with a variable path length. An end mirror $\mathrm{M}_{i}$ reflects the idler photon back to the nonlinear crystal. The optical path length of the interferometer arm for the idler, $L_{i}$, can be tuned by the position of $\mathrm{M}_{i}$ as $L_{i}=L_{i}^{0}+\Delta L$; here, $L_{i}^{0}$ is a reference point, where the optical path length of the idler matches that of the signal, $L_{i}^{0}=L_{s}$, and $\Delta L$ is the optical path length difference between the two arms of the interferometer introduced by the translation of $\mathrm{M}_{i}$. The sample whose optical properties in the infrared region are of interest is placed in the idler path. Then, the idler photon passes through the sample twice. All the pump and SPDC photons are recombined in the nonlinear crystal and the second passage of the pump light causes another photon-pair generation via SPDC. The signal photon count rate is recorded as a function of $\Delta L$ as the quantum interferogram.

We follow the approach in Refs. [20,30] to describe the generation and evolution of frequency-correlated photon pairs in the nonlinear interferometer in a low gain regime, where multiple photon emission can be neglected. For simplicity, we assume that all the SPDC photons are generated in a collinear geometry and there are no optical losses within the pump and signal paths in the interferometer. A final state for the SPDC photons is given by a superposition of two states, $|\psi\rangle_{1}$ and $|\psi\rangle_{2}$, produced by the first and second SPDC processes, respectively [Fig. 1(b)]. 
(a)

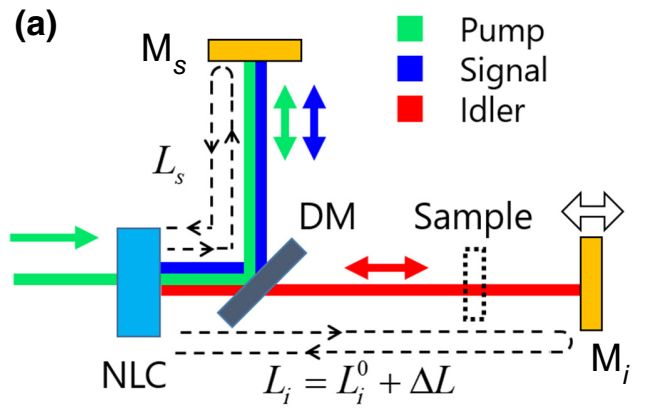

(b)

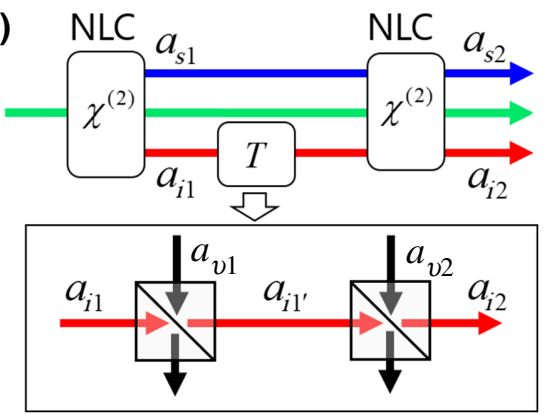

FIG. 1. (a) Schematic of nonlinear Michelson interferometer. Photons are generated via SPDC in a nonlinear crystal. NLC, nonlinear crystal with second order nonlinear susceptibility $\chi^{(2)} ; \mathrm{M}_{s, i}$, end mirrors; DM, long-pass dichroic mirror. $L_{s}$ and $L_{i}$ are the optical path lengths of the signal and idler. $L_{i}^{0}$ is the reference point, and $\Delta L$ is the optical path length difference between the interferometer arms. (b) Schematic diagram of SPDC photon-pair generation and mode conversions in the nonlinear interferometer. The pump, signal, and idler modes are indicated by green, blue, and red arrows, respectively. The lower illustration describes a splitter model, which represents the optical loss and phase retardation due to a sample inserted in the idler path. At the first beam splitter (first passage through the sample), the incident idler mode $a_{i 1}$ is converted to $a_{i 1^{\prime}}$ on being coupled with a vacuum mode $a_{v 1}$, which enters from the open port of the beam splitter. Similarly, $a_{i 1^{\prime}}$ is converted to $a_{i 2}$ on being coupled with another vacuum mode $a_{v 2}$.

$$
\begin{aligned}
|\Psi\rangle= & |\psi\rangle_{1}+|\psi\rangle_{2}=|\mathrm{vac}\rangle \\
& +\alpha \iint d \omega_{s} d \omega_{i} F\left(\omega_{s}, \omega_{i}\right) a_{s 1}^{\dagger}\left(\omega_{s}\right) a_{i 1}^{\dagger}\left(\omega_{i}\right)|\mathrm{vac}\rangle \\
& +\alpha e^{i \phi_{p}} \iint d \omega_{s} d \omega_{i} F\left(\omega_{s}, \omega_{i}\right) a_{s 2}^{\dagger}\left(\omega_{s}\right) a_{i 2}^{\dagger}\left(\omega_{i}\right)|\mathrm{vac}\rangle
\end{aligned}
$$

where $|\alpha|^{2}$ is the conversion efficiency of the SPDC processes, $F\left(\omega_{s}, \omega_{i}\right)$ is the two-photon amplitude, which defines the spectral profile of the generated photon pair [31], $a_{s 1(2)}^{\dagger}\left(\omega_{s}\right)$ and $a_{i 1(2)}^{\dagger}\left(\omega_{i}\right)$ are photon creation operators, the subindices $s 1(2)$ and $i 1(2)$ indicate spatial modes of the signal and idler generated by the first (second) SPDC process, respectively, $|\mathrm{vac}\rangle$ is the vacuum state, and $\phi_{p}$ is the phase acquired by the pump during propagation in the interferometer arm [32]. Here, the conversion efficiency $|\alpha|^{2}$ is given by $\sinh ^{2}(g z)$, where $g$ is the parametric gain (proportional to the pump field) and $z$ is the interaction length of the SPDC process [17,33]. In the low gain regime, the conversion efficiency is much lower than unity $\left(|\alpha|^{2} \ll 1\right)$. Given that the optical paths are aligned such that the spatial modes of the first SPDC photons perfectly match those of the second SPDC photon, the relation between photon creation operators for the signal (idler) for the first and second SPDC can be written as follows [Fig. 1(b)]:

$$
\begin{gathered}
a_{s 2}^{\dagger}=e^{-i \omega_{s} t_{s}} a_{s 1}^{\dagger}, \\
a_{i 2}^{\dagger}=e^{-i \omega_{i} t_{i}}\left\{\left[\tau^{*}\left(\omega_{i}\right)\right]^{2} a_{i 1}^{\dagger}+\tau^{*}\left(\omega_{i}\right) r^{*}\left(\omega_{i}\right) a_{v 1}^{\dagger}+r^{*}\left(\omega_{i}\right) a_{v 2}^{\dagger}\right\},
\end{gathered}
$$

where $t_{s(i)}$ is the propagation time of the signal (idler) photon in the interferometer arms, and the acquired phases for each mode are given by $\omega_{s} t_{s}$ and $\omega_{i} t_{i}$, respectively. In Eq. (3), the optical loss and phase retardation caused by the sample in the idler path are described by a complex transmission coefficient $\tau\left(\omega_{i}\right)$ and reflection coefficient $r\left(\omega_{i}\right)=$ $\sqrt{1-\tau^{2}\left(\omega_{i}\right)}$, using the beam splitter model [34], and $a_{v 1}$ and $a_{v 2}$ denote vacuum fields entering from open ports of the beam splitters as depicted in Fig. 1(b). The signal photon count rate is given by $P_{s}=\left\langle\Psi\left|E_{s}^{(-)} E_{s}^{(+)}\right| \Psi\right\rangle$, where $E_{s}^{(+)}(t) \propto \int d \omega a_{s 2}(\omega) e^{-i \omega t}$ is the positive frequency part of the electric field operator. From Eqs. (1)-(3), we obtain

$$
\begin{aligned}
& E_{s}^{(+)}(t)|\Psi\rangle \propto \iint d \omega_{s} d \omega_{i} e^{-i \omega_{s} t} F\left(\omega_{s}, \omega_{i}\right)\left\{\left[1+e^{i \varphi_{p}-i \omega_{s} t_{s}-i \omega_{i} t_{i}} \tau^{* 2}\left(\omega_{i}\right)\right] a_{i 1}^{\dagger}\left(\omega_{i}\right)\right. \\
& \left.+e^{i \varphi_{p}-i \omega_{s} t_{s}-i \omega_{i} t_{i}}\left[r^{*}\left(\omega_{i}\right) \tau^{*}\left(\omega_{i}\right) a_{v 1}^{\dagger}\left(\omega_{i}\right)+r^{*} a_{v 2}^{\dagger}\left(\omega_{i}\right)\right]\right\}|\mathrm{vac}\rangle .
\end{aligned}
$$

As the signal frequency is uniquely determined from the conjugate idler frequency under monochromatic excitation, the two-photon amplitude can be rewritten as $F\left(\omega_{s}, \omega_{i}\right)=F^{\prime}\left(\omega_{i}\right) \delta\left(\omega_{s}+\omega_{i}-\omega_{p}\right)$. Then, Eq. (4) can be rewritten as

$$
\begin{aligned}
E_{s}^{(+)}(t)|\Psi\rangle \propto & \iint d \omega_{s} d \omega_{i} e^{-i \omega_{\mathrm{s}} t} F^{\prime}\left(\omega_{i}\right) \delta\left(\omega_{s}+\omega_{i}-\omega_{p}\right)\left\{\left[1+e^{i \varphi_{p}-i \omega_{s} t_{s}-i \omega_{i} t_{i}} \tau^{* 2}\left(\omega_{i}\right)\right] a_{i 1}^{\dagger}\left(\omega_{i}\right)\right. \\
& \left.+e^{i \varphi_{p}-i \omega_{s} t_{s}-i \omega_{i} t_{i}}\left[r^{*}\left(\omega_{i}\right) \tau^{*}\left(\omega_{i}\right) a_{v 1}^{\dagger}\left(\omega_{i}\right)+r^{*} a_{v 2}^{\dagger}\left(\omega_{i}\right)\right]\right\}|\mathrm{vac}\rangle
\end{aligned}
$$




$$
\begin{aligned}
= & \int d \omega_{i} e^{-i\left(\omega_{p}-\omega_{i}\right) t} F^{\prime}\left(\omega_{s}\right)\left\{\left[1+e^{i \varphi_{p}-i\left(\omega_{p}-\omega_{i}\right) t_{s}-i \omega_{i} t_{i}} \tau^{* 2}\left(\omega_{i}\right)\right] a_{i 1}^{\dagger}\left(\omega_{i}\right)\right. \\
& \left.+e^{i \varphi_{p}-i\left(\omega_{p}-\omega_{i}\right) t_{s}-i \omega_{i} t_{i}}\left[r^{*}\left(\omega_{i}\right) \tau^{*}\left(\omega_{i}\right) a_{v 1}^{\dagger}\left(\omega_{i}\right)+r^{*} a_{v 2}^{\dagger}\left(\omega_{i}\right)\right]\right\}|\mathrm{vac}\rangle .
\end{aligned}
$$

The signal photon count rate is given by

$$
P_{s} \propto 2+\int_{0}^{\infty} d \omega_{i}\left|F^{\prime}\left(\omega_{i}\right)\right|^{2}\left\{\left[\tau^{*}\left(\omega_{i}\right)\right]^{2} e^{i \varphi_{p}-i\left(\omega_{p}-\omega_{i}\right) t_{s}} e^{-i \omega_{i} t_{i}}+\left[\tau\left(\omega_{i}\right)\right]^{2} e^{-i \varphi_{p}+i\left(\omega_{p}-\omega_{i}\right) t_{s}} e^{+i \omega_{i} t_{i}}\right\}
$$

where the interval of integration is indicated for the sake of clarity. Here, we used the normalization condition for the two-photon amplitude, $\int d \omega_{i}\left|F^{\prime}\left(\omega_{i}\right)\right|^{2}=1$. Equation (6) shows that the quantum interference of the photonpair generation processes results in a variation of the signal photon count rate as a function of the relative phase between the pump and SPDC photons. As the optical path lengths of the pump and signal paths are fixed, $\varphi_{p}$ and $t_{s}$ can be considered to be constant; the idler propagation time, $t_{i}$, is the only variable parameter for observing the quantum interference. Moreover, it is indicated that the visibility and phase of the interferometric signal are affected by $\tau$, which represents the infrared absorption and phase retardation caused by the sample. We note that the frequency dependence of the transmission coefficient is explicitly represented in Eq. (6), while, in Ref. [20], $\tau$ was regarded as a constant since the authors performed a wavelength-selective measurement with a narrow detection bandwidth using a monochromator, where the chromatic change in optical constants for the sample was negligibly small.

In conventional FTIR spectroscopy, interferograms are typically obtained as a function of the optical path length difference, with the wave number, $k$, used for spectral analysis. In the following sections, we show the use of the quantum interferogram in the same manner, with the Fourier spectrum given in terms of the wave number. The analytical expressions derived in this section can be easily converted to the parameter $\Delta L$, using the following relations between each set of parameters: the propagation time and path length, $t_{s}=$ $L_{s} / c, t_{i}=\left(L_{i}+\Delta L\right) / c$; the frequency and wave number, $\omega_{s(p)}=c k_{s(p)}$, where $c$ is the speed of light in vacuum. Using the parameters defined above, Eq. (6) can be rewritten as

$$
P_{s}(\Delta L) \propto 2+\int_{0}^{\infty} d k_{i}\left|F^{\prime}{ }_{k}\left(k_{i}\right)\right|^{2}\left\{\left[\tau_{k}^{*}\left(k_{i}\right)\right]^{2} e^{i \varphi_{p}-i\left(k_{p}-k_{i}\right) L_{s}-i k_{i} L_{i}^{0}} e^{-i k_{i} \Delta L}+\left[\tau_{k}\left(k_{i}\right)\right]^{2} e^{-i \varphi_{p}+i\left(k_{p}-k_{i}\right) L_{s}+i k_{i} L_{i}^{0}} e^{i k_{i} \Delta L}\right\},
$$

where $F_{k}^{\prime}(k)=F^{\prime}(c k)$ and $\tau_{k}(k)=\tau(c k)$ are the two-photon amplitude and transmission coefficients, respectively, represented as functions of $k$.

\section{B. Fourier analysis of interferogram}

In the previous section, we derive the analytical expression for the quantum interferogram. Here, we develop the theoretical basis for the Fourier analysis applicable for IRQAS. The Fourier spectral amplitude of the interferogram at wave number $k$ is defined as

$$
A_{s}(k)=\frac{1}{2 \pi} \int d \Delta L P_{s}(\Delta L) e^{i k \Delta L}
$$

By substituting Eq. (7) into the above equation and integrating, we get the following for a positive wave number, $k>0$,

$$
\begin{aligned}
A_{s}(k) & \propto \int d \Delta L \int_{0}^{\infty} d k_{i} e^{i k \Delta L}\left|F^{\prime}{ }_{k}\left(k_{i}\right)\right|^{2}\left\{\left[\tau_{k}^{*}\left(k_{i}\right)\right]^{2} e^{i \varphi_{p}-i\left(k_{p}-k_{i}\right) L_{s}-i k_{i} L_{i}^{0}} e^{-i k_{i} \Delta L}+\left[\tau_{k}\left(k_{i}\right)\right]^{2} e^{-i \varphi_{p}+i\left(k_{p}-k_{i}\right) L_{s}+i k_{i} L_{i}^{0}} e^{i k_{i} \Delta L}\right\} \\
& =\int_{0}^{\infty} d k_{i}\left|F^{\prime}{ }_{k}\left(k_{i}\right)\right|^{2}\left\{\left[\tau_{k}^{*}\left(k_{i}\right)\right]^{2} e^{i \varphi_{p}-i\left(k_{p}-k_{i}\right) L_{s}-i k_{i} L_{i}^{0}} \delta\left(k-k_{i}\right)+\left[\tau_{k}\left(k_{i}\right)\right]^{2} e^{-i \varphi_{p}+i\left(k_{p}-k_{i}\right) L_{s}+i k_{i} L_{i}^{0}} \delta\left(k+k_{i}\right)\right\} \\
& =\left|F^{\prime}{ }_{k}(k)\right|^{2}\left[\tau_{k}^{*}(k)\right]^{2} e^{i \varphi_{p}-i\left(k_{p}-k\right) L_{s}-i k L_{i}^{0}},
\end{aligned}
$$


and for a negative wave number

$$
\begin{aligned}
& A_{s}(-k) \propto \int d \Delta L \int_{0}^{\infty} d k_{i} e^{-i k \Delta L}\left|F^{\prime}{ }_{k}\left(k_{i}\right)\right|^{2}\left\{\left[\tau_{k}^{*}\left(k_{i}\right)\right]^{2} e^{i \varphi_{p}-i\left(k_{p}-k_{i}\right) L_{s}-i k_{i} L_{i}^{0}} e^{-i k_{i} \Delta L}+\left[\tau_{k}\left(k_{i}\right)\right]^{2} e^{-i \varphi_{p}+i\left(k_{p}-k_{i}\right) L_{s}+i k_{i} L_{i}^{0}} e^{i k_{i} \Delta L}\right\} \\
& =\int_{0}^{\infty} d k_{i}\left|F^{\prime}{ }_{k}\left(k_{i}\right)\right|^{2}\left\{\left[\tau_{k}^{*}\left(k_{i}\right)\right]^{2} e^{i \varphi_{p}-i\left(k_{p}-k_{i}\right) L_{s}-i k_{i} L_{i}^{0}} \delta\left(k+k_{i}\right)+\left[\tau_{k}\left(k_{i}\right)\right]^{2} e^{-i \varphi_{p}+i\left(k_{p}-k_{i}\right) L_{s}+i k_{i} L_{i}^{0}} \delta\left(k-k_{i}\right)\right\} \\
& =\left|F^{\prime}{ }_{k}(k)\right|^{2}\left[\tau_{k}(k)\right]^{2} e^{-i \varphi_{p}+i\left(k_{p}-k\right) L_{s}+i k L_{i}^{0}} \\
& =\left[A_{s}(k)\right]^{*} \text {. }
\end{aligned}
$$

Here, we have omitted an integral of the constant value, $1 /(2 \pi) \int d \Delta L(2) e^{i k \Delta L}$, which gives a zero-frequency component. To extract the information about the complex transmittance, we need to measure the quantum interferogram without a sample, $P_{s}^{0}(\Delta L)$, and calculate the Fourier spectrum, $A_{s}^{0}(k)=1 /(2 \pi) \int d \Delta L P_{s}^{0}(\Delta L) e^{i k \Delta L}$. Clearly, $A_{s}^{0}(k)$ is given by setting the value of the transmission coefficient in Eq. (9) to 1. The ratio of the complex Fourier spectra directly gives the complex transmittance,

$$
A_{s}(k) / A_{s}^{0}(k)=\left[\tau_{k}^{*}(k)\right]^{2} .
$$

Note that the total transmission coefficient, $T=\tau^{2}$, described by Eq. (11), takes into account the double passage of the idler photon through the sample. The transmission coefficient for a single passage can be obtained by taking the square root of Eq. (11).

Once the complex transmittance at each frequency is determined, we can directly calculate the complex-valued optical constants for the sample. Following the conventional procedures of time-domain spectroscopy, we consider the extraction of the refractive index for a slab sample in a transmission geometry. For normal incidence of a photon with wave number $k$ into a slab sample with thickness $d$ and complex refractive index $N=n+i \kappa$, where $n$ and $\kappa$ are real and imaginary part of refractive index, respectively, $T(k)$ for a double passage can be written as

$$
T(k)=\left\{\frac{4 N(k)}{[1+N(k)]^{2}}\right\}^{2} e^{i 4 \pi k d[N(k)-1]} .
$$

In this equation, the exponential term describes the optical absorption and phase retardation during the passage inside the slab sample. Substituting the experimentally obtained values of $T(k)$, we can determine $N(k)$ from Eq. (12). In the next section, we describe the experimental realization of a QFTIR spectroscopy system and demonstrate the refractive index measurement for a sample.

\section{EXPERIMENTAL SETUP}

Figure 2 depicts our experimental setup. As we considered in the previous section, the quantum interferogram is obtained with a nonlinear Michelson interferometer based on a SPDC photon-pair source. A continuous wave (cw) laser with a wavelength of $532 \mathrm{~nm}$ (power $150 \mathrm{~mW}$, linewidth $<1 \mathrm{MHz}$ ) is used as a pump. The pump light is focused by a lens L1 $(f=150 \mathrm{~mm})$ into a nonlinear optical crystal, and reflected by a dichroic mirror DM1. The pump beam diameter is $2 \mathrm{~mm}$ before the focusing lens L1. For the nonlinear crystal, we chose a $5-\mathrm{mol} \% \mathrm{MgO}$-doped $\mathrm{LiNbO}_{3}$ (LN) crystal with a thickness of $0.5 \mathrm{~mm}$. We utilize the type-I $(e \rightarrow o o$ ) collinear SPDC process for visibleinfrared photon-pair generation, where the pump light with extraordinary polarization $(e)$ is down-converted into signal and idler photons with ordinary polarizations $(o)$. The polarization of the pump is rotated in the appropriate direction for this SPDC condition by a half-wave plate, HWP. The SPDC wavelength of the infrared idler can be selectively tuned over a wide spectral range up to $4700 \mathrm{~nm}$ by mechanical adjustment of the propagation angle of the pump beam against the optic axis in the LN crystal [35]. The propagation angle is set at around $70^{\circ}$, so that the SPDC wavelengths are phase matched at around $810 \mathrm{~nm}$ (signal) and $1550 \mathrm{~nm}$ (idler).

After the first SPDC process, the pump and signal are reflected by a dichroic mirror, DM2, and refocused into the $\mathrm{LN}$ crystal by a concave end mirror, $\mathrm{CM}(f=100 \mathrm{~mm})$. The idler transmitted by DM2 is collimated by a lens, L2 $(f=100 \mathrm{~mm})$, and propagated along an interferometer arm. An end mirror, $\mathrm{M}_{i}$, is placed on a translational stage (FS-1050UPX, Sigma Koki), which acts as a highresolution feedback stage with a position repeatability of $\pm 2 \mathrm{~nm}$. The optical path length, and equivalently the propagation time for the idler, can be tuned by a translational shift in the position of $\mathrm{M}_{i}$. The sample is placed between $\mathrm{L} 2$ and $\mathrm{M}_{i}$ in the idler arm, then the idler photon passes through the sample twice. The idler photon is also refocused into the crystal and recombined with the pump and signal. The second passage of the pump causes another photon-pair generation. As explained in the previous section, the total photon-pair emission rate depends on the relative phase between all the photons related to the consecutive SPDC processes. The visible signal photon leaving the nonlinear interferometer passes through 


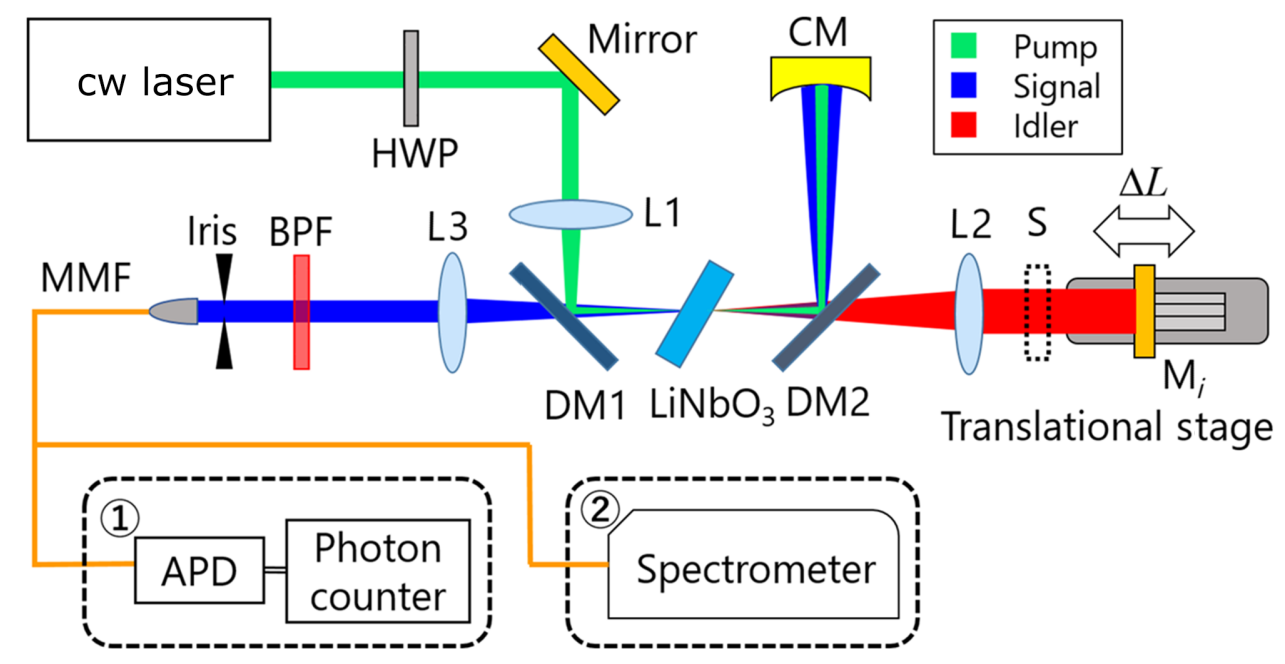

FIG. 2. Schematic of the experimental setup. HWP, half wave plate; L1, focusing lens for the pump ( $f=150 \mathrm{~mm})$; L2, collimation lens for the idler $(f=100 \mathrm{~mm})$; L3, collimation lens for the signal $(f=150 \mathrm{~mm})$; DM1, long-pass dichroic mirror (edge wavelength $600 \mathrm{~nm}$ ); DM2, long-pass dichroic mirror (edge wavelength $1000 \mathrm{~nm}$ ); CM, concave end mirror; $\mathrm{M}_{i}$, end mirror; BPF, bandpass filter (center wavelength $850 \mathrm{~nm}$, bandwidth $50 \mathrm{~nm}$ ); MMF, multimode fiber; APD, Si avalanche photodiode; S, sample (silica glass plate). A continuous wave (cw) laser with a wavelength of $532 \mathrm{~nm}$ (power $150 \mathrm{~mW}$, linewidth $<1 \mathrm{MHz}$ ) is used as a pump.

a dichroic mirror, DM1, and is collimated by a lens, L3 $(f=150 \mathrm{~mm})$, placed after DM1. A bandpass filter, BPF, transparent to the signal wavelength, filters out unwanted spectral components, such as the residual pump transmitted by DM1 and luminescence from the LN crystal; the bandwidth of BPF is wider than the spectral width of the signal emission. If necessary, an iris is placed after L3 to spatially select only the center-most part of the signal beam where the SPDC emission angle is close to perfect collinear geometry.

The signal photons are coupled to a multimode fiber, $\mathrm{MMF}$, and sent to a detection system for quantum interferogram measurement (dashed box 1 in Fig. 2). The flux of the signal photons is measured by a single photon counting avalanche photodiode, APD (SPCM-AQRH14FC, Excelitas Tech.), and a photon counter (SR400, Stanford Research System). The signal photon count rate, $P_{s}$, is recorded as a function of $\Delta L$, translating $\mathrm{M}_{i}$ in the idler path. From the obtained quantum interferograms, measured with and without a sample, we calculate the Fourier spectra and extract the complex transmittance according to Eqs. (8)-(11). The signal photon is also sent to a dispersive spectrometer with a CCD array detector (SR500i + DU416A-LDC-DD, Andor, with wavelength resolution $0.1 \mathrm{~nm}$, shown in dashed box 2 in Fig. 2) to measure the spectral profile of the signal emission. From the obtained signal spectrum and energy correlation of the SPDC photon pair, we can infer the center wavelength and bandwidth of the infrared idler spectrum.

Note that we used the cw laser for pumping the crystals. In this case, the conversion efficiency $|\alpha|^{2}$ is given by the number of generated photon pairs per the correlation time of biphotons, which is about 90 fs. The number of generated photon pairs from the crystal is estimated to be at most $4.7 \times 10^{7}$ pairs $/ \mathrm{s}$ for the pump intensity of $150 \mathrm{~mW}$, considering the count rate of signal photons $2.8 \times 10^{6}$ counts $/ \mathrm{s}$ and the effective total detection efficiency of $6 \%$ (the quantum efficiency of the APD is $60 \%$ and the coupling efficiency of photons to the multimode fiber including optical loss in the system is at least 10\%). As a result, the estimated number of photon pairs per the biphotons' correlation time $|\alpha|^{2}$ is $4.2 \times 10^{-6}$ and $|\alpha|$, which is approximately equal to $g z$, is $2.1 \times 10^{-3}$, which is much less than 1 . Thus, we can conclude that our experiment shown in the next section is performed in the low gain regime.

\section{RESULTS AND DISCUSSION}

\section{A. Observation of quantum interferogram}

First, we measure the quantum interferogram without a sample. The angle of the LN crystal is adjusted such that the signal and idler wavelengths are phase matched at around 809 and $1554 \mathrm{~nm}$, respectively. The aperture of the iris in the signal path is set to $1.5 \mathrm{~mm}$. The signal photon count rate measured by the APD is shown in Fig. 3(a) as a function of $\Delta L$. We translate $\mathrm{M}_{i}$ with step sizes of $50 \mathrm{~nm}$ (equivalent to a change in $\Delta L$ of $100 \mathrm{~nm}$ ) up to a total scanning length, $W$, of $200 \mu \mathrm{m}$. The photon count is integrated over $300 \mathrm{~ms}$ for each step. The inset of Fig. 3(a) is an expanded plot of the same data around $\Delta L=0$. The origin of the optical path length difference is defined as the position where the interference fringe takes the maximum value. The periodicity of the interferogram is estimated to 

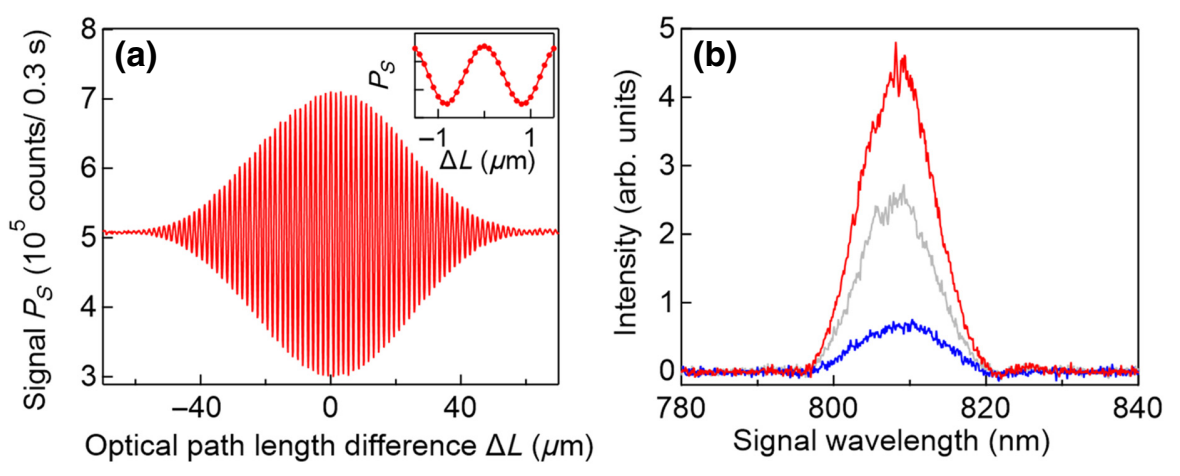

FIG. 3. (a) Quantum interferogram with signal and idler wavelengths of 809 and $1554 \mathrm{~nm}$, respectively. The inset shows the expanded plot around $\Delta L=0 \mathrm{~nm}$. (b) Signal emission spectra measured with $\Delta L=0 \mathrm{~nm}$ is denoted by the red line, and $800 \mathrm{~nm}$, denoted by the blue line. The light gray line shows an emission spectrum measured with the quantum interference suppressed by blocking the idler path. be around $1550 \mathrm{~nm}$, as expected from Eq. (7), coincident with the center wavelength of the idler.

We also investigate how quantum interference affects the signal photon generation in the spectral domain. In Fig. 3(b), the light gray plot shows the signal emission spectrum when the quantum interference of the SPDC processes is completely suppressed by blocking the idler photon generated via the first SPDC (the $i 1$ mode photon). To remove unwanted fluorescence from the LN crystal and stray light, we perform a background measurement, rotating the pump light polarization by $90^{\circ}$ to suppress the SPDC emission, and subtract the background spectrum from each measurement of the signal spectra. The center wavelength of the signal is located at $809 \mathrm{~nm}$ and the bandwidth (full width of half maximum) is about $10 \mathrm{~nm}$. Next, we introduce the $i 1$ mode idler photon into the nonlinear crystal to observe the quantum interference. Then, the signal emission spectra are measured at $\Delta L=0 \mathrm{~nm}$ (red line) and $800 \mathrm{~nm}$ (blue line), which approximately correspond to the bright and dark fringe positions in the interferogram [Fig. 3(a)], respectively. It is clearly seen that the whole signal spectrum is drastically modulated depending on $\Delta L$. At the center wavelength, the visibility of the spectral modulation reaches $80 \%$. This result indicates that all the spectral components of the signal photon, ranging from 800 to $820 \mathrm{~nm}$, are involved in the nonlinear interference, and the spectral coverage of the IRQAS can be determined from the bandwidth of the measured signal emission spectrum, which is expected to be much larger than $100 \mathrm{~cm}^{-1}$ in wave number units.

\section{B. Transmittance measurement using QFTIR spectroscopy}

Next, to demonstrate a transmittance measurement by QFTIR spectroscopy, we measure the transmittance of a bandpass filter (BFH1550-12, Thorlabs, with a center wavelength of $1550 \mathrm{~nm}$ and bandwidth of $12 \mathrm{~nm}$ ). In these measurements, the step size is set to $100 \mathrm{~nm}$ (equivalent to a $200 \mathrm{~nm}$ step of $\Delta L$ ) and $W$ is set to 2000 and $200 \mu \mathrm{m}$ for the signals with and without the sample, respectively. The photon count is integrated over $50 \mathrm{~ms}$ for each step. The sample is inserted in the idler path. Figure 4(a) shows interferograms taken with and without the sample, $P_{s}(\Delta L)$ and $P_{s}^{0}(\Delta L)$. The peak position of the interferogram with the sample (sample signal) is shifted by about $2000 \mu \mathrm{m}$ compared to that without the sample due to phase retardation. Discrete Fourier transforms (DFTs) of the interferograms are performed based on the fast Fourier transform algorithm. To perform Fourier transforms of the signals in the same transform window, the signal without the sample is extended to a total data length
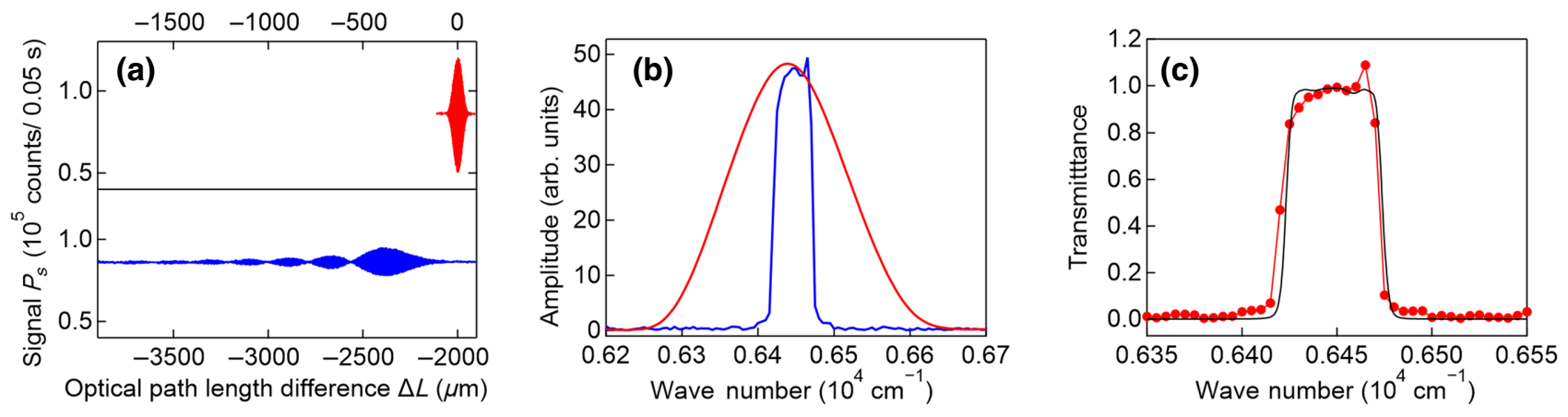

FIG. 4. (a) Waveforms and (b) magnitudes of Fourier spectra of quantum interferograms with and without the sample (bandpass filter, BFH1550-12, Thorlabs) denoted by red and blue lines, respectively. (c) Magnitude of transmittance of the sample measured by QFTIR spectroscopy (solid red circle) and measured by conventional dispersive spectrometer (UV-3600 Plus, Shimadzu Corp.) (black line). 

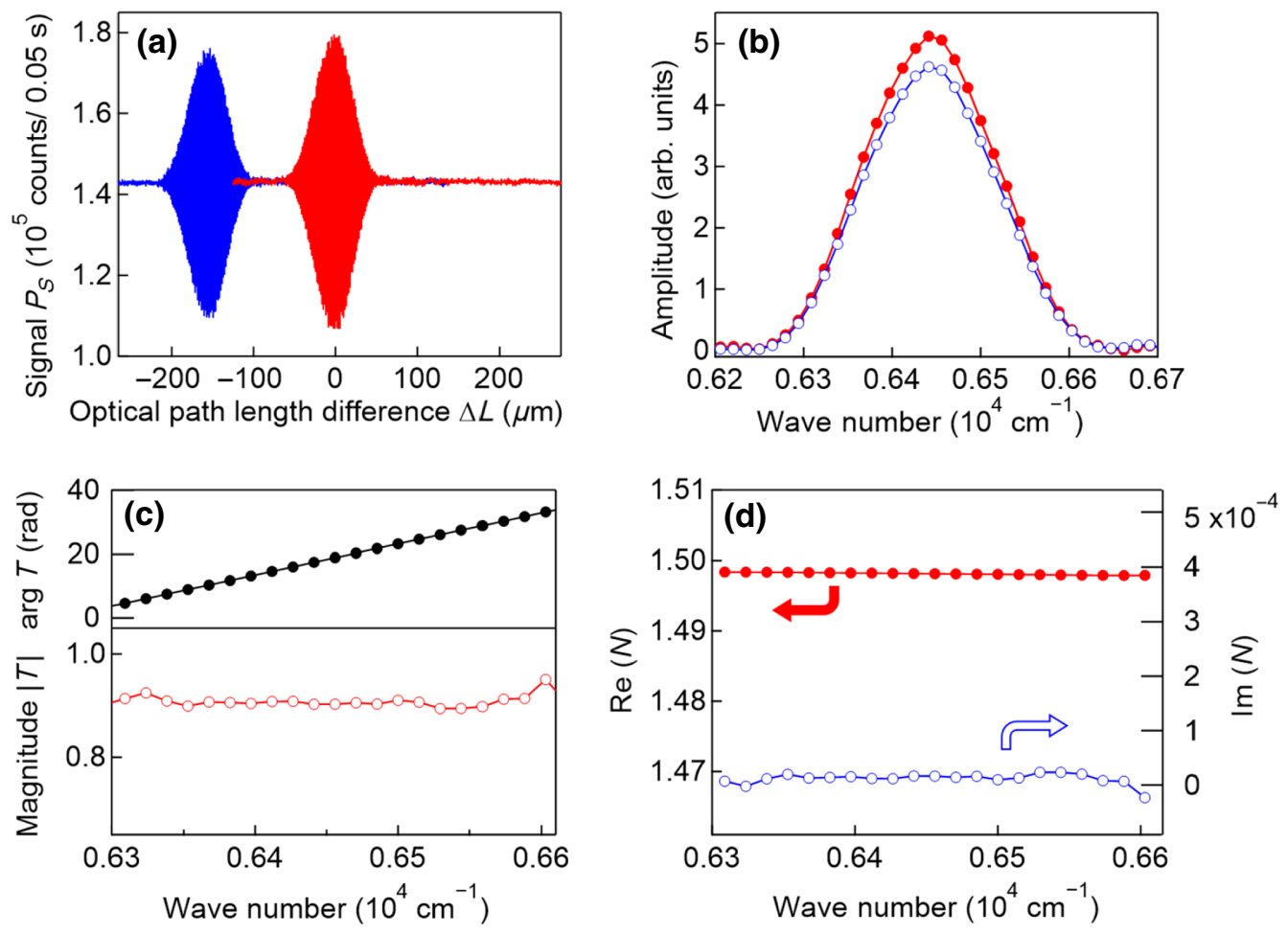

FIG. 5. (a) Quantum interferograms measured without the sample denoted by red line, and with the sample, $158-\mu \mathrm{m}$-thick silica glass denoted by blue line. (b) Magnitudes of complex Fourier amplitude spectra of the interferogram without the sample (solid red circle) and with the sample (open blue circle). (c) Phase retardation (upper panel) and magnitude of the transmittance (lower panel) of the sample. (d) Complex refractive index for the sample calculated from the transmittance data in (c). Real and imaginary parts of the complex refractive index are denoted by solid red circles and open blue circles, respectively. of $2000 \mu \mathrm{m}$ by padding additional data points. To extrapolate the dc offset of the signal, the value of the padded data is determined by the average of the signal data (without the sample) over a scanning range of $\Delta L=-100$ to $-70 \mu \mathrm{m}$. In a DFT, the wave number resolution $\Delta k$ is determined by the inverse of the width of the transform window, that is, $\Delta k=1 / W=5 \mathrm{~cm}^{-1}$. The magnitudes of the obtained complex Fourier amplitudes, $\left|A_{s}\right|$ and $\left|A_{s}^{0}\right|$, are plotted in Fig. 4(b). The center wave number of the spectrum without the sample is located around $6435 \mathrm{~cm}^{-1}$, corresponding to an idler wavelength of $1554 \mathrm{~nm}$, and the detection band spans from 6300 to $6600 \mathrm{~cm}^{-1}$. Figure 4(c) shows the transmittance of the sample obtained by taking the ratio of $\left|A_{s}\right|$ to $\left|A_{s}^{0}\right|$, which is in good agreement with a transmittance spectrum measured by a conventional dispersive spectrometer (UV-3600 Plus, Shimadzu Corp.) in terms of the magnitude and bandwidth.

\section{Complex transmittance measurement and estimation of complex refractive index using QFTIR spectroscopy}

Finally, we experimentally demonstrate that QFTIR spectroscopy enables complex transmittance measurement and the extraction of complex-valued optical constants. We apply the analytical method involving the phase information described in the previous section to determine the complex refractive index for a sample of silica glass (thickness $158 \pm 4 \mu \mathrm{m}$; the error is the standard deviation of the thickness measurements). The iris in the signal path is fully open and there is no spatial selection of the signal modes in the following measurements. Figures 5(a) and 5(b) show the waveforms and magnitudes, respectively, of Fourier spectra of the interferograms taken with and without the sample. Here, the step size is set to $100 \mathrm{~nm}$ (equivalent to a $200 \mathrm{~nm}$ step of $\Delta L$ ) and $W$ is $400 \mu \mathrm{m}$. The photon count is integrated over $50 \mathrm{~ms}$ for each step. As shown in Fig. 5(a), the peak position of the interferogram with the sample (blue line) is shifted by approximately $150 \mu \mathrm{m}$ compared to that without the sample (red line), due to the phase retardation caused by the sample. To measure the whole waveform of the interferogram within the scanning length of $400 \mu \mathrm{m}$, the start position of the $\Delta L$ scan for the sample signal is shifted by $140 \mu \mathrm{m}$. To perform Fourier transforms of the signals in the same transform window so that the spectra share a common phase origin, the interferogram signals are extended to a total data length, $W^{\prime}$, of $680 \mu \mathrm{m}$ by padding additional data points. The value of the padded data is determined by the average of the signal data (without the sample) over a scanning range of $\Delta L=145$ to $275 \mu \mathrm{m}$. The magnitudes of the obtained complex Fourier amplitudes are plotted in Fig. 5(b). Note that the wave number resolution is essentially determined by the original data span $(400 \mu \mathrm{m})$, that is, $\Delta k=1 / W=25 \mathrm{~cm}^{-1}$, while the data spacing in the spectral plots [Fig. 5(b)] is a bit finer because of the data padding, $\Delta k^{\prime}=1 / W^{\prime}=14.7 \mathrm{~cm}^{-1}$. From the obtained Fourier spectra, we calculate the complex transmittance, $T=\tau^{2}$, according to Eq. (11). Figure 5(c) shows the magnitude of the complex transmittance, $|T|$, and the phase retardance, $\arg T$. Since the phase values 
for each complex Fourier amplitude, $\arg A_{s}$ and $\arg A_{s}^{0}$, are given in the regions $-\pi<\arg A_{s}, \arg A_{s}^{0}<\pi, \arg T=$ $\arg A_{s}-\arg A_{s}^{0}$ is also given as a wrapped phase spectrum, $-2 \pi<\arg T<2 \pi$. We unwrap the spectrum as a monotonically increasing function by adding integer multiples of $2 / \pi$ to (or subtracting from) the original data. Figure 5(d) shows the refractive index $N(k)$ for the silica glass plate from the measured complex transmittance [Fig. 5(c)] and Eq. (12). At the peak wave number of the Fourier spectra, $k=6440 \mathrm{~cm}^{-1}(1550 \mathrm{~nm})$, the real part of $N$ is estimated to be $1.50 \pm 0.02$, which is in good agreement with the literature value (1.506 at $1550 \mathrm{~nm})$ [36], and the imaginary part is $1.9(+0.1 /-0.4) \times 10^{-5}$, which is fairly close to zero as expected from the fact that the silica glass is transparent in this spectral region. Here, the errors in $N$ are estimated by taking into account the uncertainty of the sample thickness $d(158 \pm 4 \mu \mathrm{m})$. It should be mentioned that, while spectral coverage in a single measurement is limited to $300 \mathrm{~cm}^{-1}$, determined by the bandwidth of the SPDC emission from the nonlinear crystal, the center wavelength of the detection photon can be tuned over a wide spectral region; for example, in the case of $\mathrm{LiNbO}_{3}$, the detection wavelength can be extended up to around $4.7 \mu \mathrm{m}$ [35].

\section{CONCLUSION}

In conclusion, we have developed the theoretical framework for QFTIR spectroscopy and experimentally confirm the validity of the method. The analysis method allows us to measure the complex transmittance of a sample and calculate the complex-valued optical constants without using a Kramers-Kronig transformation. Using a nonlinear Michelson interferometer with an SPDC-based visible-infrared photon-pair source, we observe a quantum interferogram with high visibility spectrum modulation, reaching $80 \%$ at a signal wavelength of $809 \mathrm{~nm}$. We have demonstrated the transmittance measurement of a bandpass filter by QFTIR spectroscopy in the near-infrared region around $1550 \mathrm{~nm}\left(6440 \mathrm{~cm}^{-1}\right)$, and have found that the obtained transmission spectrum is in good agreement with that measured with a conventional dispersive spectrometer. Finally, we have performed a complex transmittance measurement of silica glass and calculate its complex refractive index, fully utilizing the phase information of the quantum interferogram. The obtained value for the refractive index coincides with the literature value. The spectral coverage of QFTIR spectroscopy is estimated to be larger than $300 \mathrm{~cm}^{-1}$ and a typical value of the spectral resolution is $25 \mathrm{~cm}^{-1}$ in our setup. Owing to the multiplexing nature of Fourier transform spectroscopy, QFTIR spectroscopy does not require dispersive optics and array detectors. Since the IR light source and the IR detector have been the obstacles for the miniaturization of the conventional FTIR, QFTIR requiring only a visible light source and detectors without dispersive optics has a strong potential as a compact and cost-effective IR spectroscopy system.

The detection bandwidth can be significantly improved, combined with a broadband photon-pair source [37,38]. The operating wavelength of QFTIR spectroscopy could be extended to the mid- and far-infrared regions by choosing a suitable nonlinear crystal as the photon-pair source [39-42]. Our method can be applied to a variety of quantum spectroscopy systems, such as those based on a Mach-Zehnder nonlinear interferometer, with minor modifications, and is also applicable for infrared hyperspectral imaging [43].

It may also be interesting to explore the quantum to classical transition of the proposed optical system when the nonlinear gain is increased. Our simple analysis based on Ref. [17] suggests that in the high gain regime $(g z \gg 1)$, the visibility $V(T)$ of the interference converges to that predicted using classical theory, but $V(T)$ shows nonlinear behavior and the slope of $V(T)$ at the optical transmittance $T=1$ is 0 , suggesting that the interferometer is insensitive to a small absorption, which is in clear contrast to our QFTIR where $V(T)$ is linear between $T=0$ and 1. [As Eq. (6) indicates, the visibility of the quantum interferogram $P_{s}$ is linearly dependent on the transmittance $|T|=|\tau|^{2}$ in the low gain regime.] However, the details of the gain dependence of the sensitivity is beyond the scope of this paper and left for future investigation.

\section{ACKNOWLEDGMENTS}

The authors are grateful to K. Uchida and K. Tanaka for the measurement using the conventional dispersive interferometer. This work is supported by the MEXT Quantum Leap Flagship Program (MEXT Q-LEAP) Grant No. JPMXS0118067634, JST-CREST (JPMJCR1674), JSTPRESTO (JPMJPR15P4), Grant-in-Aid for JSPS Fellows 20J23408, and WISE Program, MEXT.

[1] P. R. Griffiths and J. A. de Haseth, Fourier Transform Infrared Spectrometry, 2nd ed. (John Wiley \& Sons, New York, 2007).

[2] S. Takeuchi, Recent progress in single-photon and entangled-photon generation and applications, Jpn. J. Appl. Phys. 53, 030101 (2014).

[3] D. Bouwmeester, J.-W. Pan, K. Mattle, M. Eibl, H. Weinfurter, and A. Zeilinger, Experimental quantum teleportation, Nature 390, 575 (1997).

[4] N. Gisin and R. Thew, Quantum communication, Nat. Photonics 1, 165 (2007).

[5] J. L. O'Brien, A. Furusawa, and J. Vučković, Photonic quantum technologies, Nat. Photonics 3, 687 (2009).

[6] R. Okamoto, J. L. O’Brien, H. F. Hofmann, T. Nagata, K. Sasaki, and S. Takeuchi, An entanglement filter, Science 323, 483 (2009). 
[7] R. Okamoto, J. L. O'Brien, H. F. Hofmann, and S. Takeuchi, Realization of a Knill-Laflamme-Milburn controlled-NOT photonic quantum circuit combining effective optical nonlinearities, Proc. Natl. Acad. Sci. 108, 10067 (2011).

[8] A. F. Abouraddy, M. B. Nasr, B. E. A. Saleh, A. V. Sergienko, and M. C. Teich, Quantum-optical coherence tomography with dispersion cancellation, Phys. Rev. A 65 , 053817 (2002).

[9] T. Nagata, R. Okamoto, J. L. O’Brien, K. Sasaki, and S. Takeuchi, Beating the standard quantum limit with fourentangled photons, Science 316, 726 (2007).

[10] V. Giovannetti, S. Lloyd, and L. Maccone, Advances in quantum metrology, Nat. Photonics 5, 222 (2011).

[11] T. Ono, R. Okamoto, and S. Takeuchi, An entanglementenhanced microscope, Nat. Commun. 4, 2426 (2013).

[12] G. B. Lemos, V. Borish, G. D. Cole, S. Ramelow, R. Lapkiewicz, and A. Zeilinger, Quantum imaging with undetected photons, Nature 512, 409 (2014).

[13] M. Okano, H. Lim, R. Okamoto, N. Nishizawa, S. Kurimura, and S. Takeuchi, $0.54 \mu \mathrm{m}$ resolution two-photon interference with dispersion cancellation for quantum optical coherence tomography, Sci. Rep. 5, 18042 (2016).

[14] R. Okamoto, Y. Tokami, and S. Takeuchi, Loss tolerant quantum absorption measurement, New J. Phys. 22, 103016 (2020).

[15] A. Kuzmich and L. Mandel, Sub-shot-noise interferometric measurements with two-photon states, Quantum Semiclassical Opt. 10, 493 (1998).

[16] M. V. Chekhova and Z. Y. Ou, Nonlinear interferometers in quantum optics, Adv. Opt. Photon. 8, 104 (2016).

[17] R. Z. Vered, Y. Shaked, Y. Ben-Or, M. Rosenbluh, and A. Pe'er, Classical-to-quantum Transition with Broadband Four-Wave Mixing, Phys. Rev. Lett. 114, 063902 (2015).

[18] Z. Y. Ou and X. Li, Quantum SU $(1,1)$ interferometers: Basic principles and applications, APL Photonics 5, 080902 (2020).

[19] D. A. Kalashnikov, A. V. Paterova, S. P. Kulik, and L. A. Krivitsky, Infrared spectroscopy with visible light, Nat. Photonics 10, 98 (2016).

[20] A. Paterova, H. Yang, Ch. An, D. Kalashnikov, and L. Krivitsky, Measurement of infrared optical constants with visible photons, New J. Phys. 20, 043015 (2018).

[21] X. Y. Zou, L. J. Wang, and L. Mandel, Induced Coherence and Indistinguishability in Optical Interference, Phys. Rev. Lett. 67, 318 (1991).

[22] L. J. Wang, X. Y. Zou, and L. Mandel, Induced coherence without induced emission, Phys. Rev. A 44, 4614 (1991).

[23] C. K. Hong and L. Mandel, Theory of parametric frequency down conversion of light, Phys. Rev. A 31, 2409 (1985).

[24] C. Lindner, S. Wolf, J. Kiessling, and F. Kühnemann, Fourier transform infrared spectroscopy with visible light, Opt. Express 28, 4426 (2020).

[25] P. Jacquinot, New developments in interference spectroscopy, Rep. Prog. Phys. 23, 267 (1960).

[26] J. Connes and P. Connes, Near-infrared planetary spectra by Fourier spectroscopy. I. Instruments and results, J. Opt. Soc. Am. 56, 896 (1966).
[27] T. D. Dorney, R. G. Baraniuk, and D. M. Mittleman, Material parameter estimation with terahertz time-domain spectroscopy, J. Opt. Soc. Am. A 18, 1562 (2001).

[28] P. U. Jepsen, D. G. Cooke, and M. Koch, Terahertz spectroscopy and imaging - modern techniques and applications, Laser Photon. Rev. 5, 124 (2011).

[29] Y. R. Shen, The Principles of Nonlinear Optics (Wiley, New York, 1984).

[30] M. Lahiri, R. Lapkiewicz, G. B. Lemos, and A. Zeilinger, Theory of quantum imaging with undetected photons, Phys. Rev. A 92, 013832 (2015).

[31] A. V. Burlakov, M. V. Chekhova, D. N. Klyshko, S. P. Kulik, A. N. Penin, Y. H. Shih, and D. V. Strekalov, Interference effects in spontaneous two-photon parametric scattering from two macroscopic regions, Phys. Rev. A 56, 3214 (1997).

[32] A. Heuer, S. Raabe, and R. Menzel, Phase memory across two single-photon interferometers including wavelength conversion, Phys. Rev. A 90, 045803 (2014).

[33] A. Yariv, Quantum Electronics (John Wiley \& Sons, New York, 1988).

[34] L. Mandel and E. Wolf, Optical Coherence and Quantum Optics (Cambridge University Press, New York, 1995).

[35] M. Arahata, B. Cao, T. Tashima, Y. Mukai, R. Okamoto, and S. Takeuchi, in Proc. The 2nd International Forum on Quantum Metrology and Sensing (IFQMS), B-25 (2019).

[36] SCHOTT glass database (D263 ${ }^{\mathrm{R}} \mathrm{T}$ eco). http://www.schott. com; https://refractiveindex.info/download/data/2017/ schott_2017-01-20b.agf.

[37] A. Tanaka, R. Okamoto, H.-H. Lim, S. Subashchandran, M. Okano, L. Zhang, L. Kang, J. Chen, P. Wu, T. Hirohata, S. Kurimura, and S. Takeuchi, Noncollinear parametric fluorescence by chirped quasi-phase matching for monocycle temporal entanglement, Opt. Express 20, 25228 (2012).

[38] K. Sugiura, Z. Yin, R. Okamoto, L. Zhang, L. Kang, J. Chen, P. Wu, S. T. Chu, B. E. Little, and S. Takeuchi, Broadband generation of photon-pairs from a CMOS compatible device, Appl. Phys. Lett. 116, 224001 (2020).

[39] Y. M. Sua, H. Fan, A. Shahverdi, J.-Y. Chen, and Y.-P. Huang, Direct generation and detection of quantum correlated photons with $3.2 \mu \mathrm{m}$ wavelength spacing, Sci. Rep. 7, 17494 (2017).

[40] B. Haase, M. Kutas, F. Riexinger, P. Bickert, A. Keil, D. Molter, M. Bortz, and G. von Freymann, Spontaneous parametric down-conversion of photons at $660 \mathrm{~nm}$ to the terahertz and sub-terahertz frequency range, Opt. Express 27, 7458 (2019).

[41] S. Prabhakar, T. Shields, A. C. Dada, M. Ebrahim, G. G. Taylor, D. Morozov, K. Erotokritou, S. Miki, M. Yabuno, H. Terai, C. Gawith, M. Kues, L. Caspani, R. H. Hadfield, and M. Clerici, Two-photon quantum interference and entanglement at $2.1 \mu \mathrm{m}$, Sci. Adv. 6, eaay5195 (2020).

[42] M. Kutas, B. Haase, P. Bickert, F. Riexinger, D. Molter, and G. von Freymann, Terahertz quantum sensing, Sci. Adv. 6, eaaz8065 (2020).

[43] I. Kviatkovsky, H. M. Chrzanowski, E. G. Avery, H. Bartolomaeus, and S. Ramelow, Microscopy with undetected photons in the mid-infrared, arXiv:2002.05960 (2019). 